\title{
PROGRESSIVE CHANGE DETECTION IN TIME SERIES OF SAR IMAGES
}

\author{
Grégoire Mercier
}

\author{
Institut Telecom; Telecom Bretagne \\ CNRS UMR 3192 lab-STICC, team CID \\ Technopole Brest-Iroise, \\ CS 83818, F-29238 Brest Cedex 3, France
}

\begin{abstract}
The aim of this paper is to present a general framework for change detection in a time series of radar images, for an operational purpose and in the context of environmental monitoring. The change detection procedure is turned into the framework of detecting a random signal into the noise; the detection of this signal leads to the detection of a change in the time series. This framework is based on a non-parametric detection method that assume a sparse representation of the data. When using radar images, the speckle noise invalidates the hypothesis of sparsity. Then a pre-processing technique is required to provide highly sparsifiable representation of data, whatever the initial noise characteristics.

The paper focuses on the change indicator, based on recursive median filtering, yielding a piecewise regular representation of a scene obtained by spreading the statistically most reliable pixel values over the image.

The recursive median filtering leads to simple change indicators that are more efficient than the Kullback-Leibler change indicator when using small analysing sliding window. Furthermore, it induces an simple extension to perform progressive change characterization through a multitemporal filtering approach.

Results are shown with a two-date change detection from RADARSAT images and from a time series of ERS and ENVISAT images.
\end{abstract}

\section{THE PROPOSED METHODOLOGY}

\subsection{Introduction}

The change detection method proposed in this paper involves conditioning the SAR data in order to reduce the variability induced by speckle noise and mapping the change detection problem, as defined in [1], into a standard problem of signal detection in noisy observations. Conditioning the data is achieved by using an algorithm based on the local statistics of the data. This algorithm performs a regularization of the signal which reduces the high variability that characterizes the SAR data without impacting significantly the change information. By reducing SAR data variability with this algorithm, the difference between multitemporal SAR observations is shown to be sufficiently sparse for detecting the change signal by evaluating the amplitudes of the data, when the changes affect a certain region of the scene and the change signal being dense in this region. Change detection is then mapped into a standard problem of signal detection in noisy observations and a method for selecting the threshold level is also proposed for differencing the changed and unchanged data. At this step, it does not intend to charcterize the type of changes that are detected, as it has been proposed in [2]. The change detection method developped in this paper is of low computational complexity and proved its effectiveness on medium resolution SAR images.

\subsection{Problem Formulation}

Let $\boldsymbol{I}$ be a sequence of $M+1$ co-registred SAR images acquired at time $\left\{t_{0}, t_{1}, \ldots, t_{M}\right\}: \boldsymbol{I}=\left\{I_{t}, t=t_{0}, t_{1}, \ldots, t_{M}\right\}$. Let $I_{t}^{(i, j)}$ be the pixel of image $I_{t}$ at position $(i, j)$. In this study, it is assumed that a change map is a sparse map with the same size as $I_{0}$ and which contains the change areas detected by comparing the different observations. In practice, this change map is sparse when the images are acquired before and after a change event, that is when the images under consideration contain both changed and non-changed areas.

It is assumed that images are processed so that the difference between two images is turned to be significantly piecewise regular in presence of changes. Then the change measure is set to be the amplitude of the difference operator between the images. 


\subsection{Overview of the proposed method}

The following describes the steps used for the change detection algorithm proposed in this paper. Each of these steps involves a small computation time so that it makes the overall method adapted for operational use.

(i) Image regularisation processing consists in conditionning the data in order to make the absolute difference between observations more piecewise regular, that is, more reliable for the change detection purpose. Let consider a 1D signal $\boldsymbol{x}$ and a sequence of $2 r+1$ sample around $x_{i}$, denoted to as $x_{[i ; r]}$ :

$$
x_{i-r}, \ldots, x_{i-1}, x_{i}, x_{i+1}, \ldots, x_{i+r} .
$$

$x_{i}$ may be filtered by considering the following measure of the information in the sequence $x_{[i ; r]}$. The Recursive Median (RM) principle leads to [3]

$$
y_{i}=\operatorname{median}\left(y_{i-r}, \ldots, y_{i-1}, x_{i}, x_{i+1}, \ldots, x_{i+r}\right) .
$$

The extension to image (i.e. 2D signal) processing is not straingforward. An Peano-Hilbert scan has been used to go back to the 1D RM formulation. It prevents from possible hysteresis effects which can occur in the neighborhood of edges. Denote by $\mathcal{I}_{t}$, the result of the recursive median filtering of $I_{t}$.

(ii) Change measure computation by combining (differency) two-by-two regularised observations in the time series. The twoby-two change measurements can be obtained from consecutive acquisitions and fits 2-date abrupt change detection. The change measurement can also be composed by a set of two-by-two change measurement so that it fits progressive change characterization point of view. Bi-date change measurement is performed by considering the absolute difference between pre-processed observations. At a given location $(i, j)$, the change amplitude between two observations acquired at times $t_{\ell}$ and $t_{\ell+1}$, respectively, is computed by

$$
\delta^{(i, j)}\left(t_{\ell}, t_{\ell+1}\right)=\left|\mathcal{I}_{t_{\ell}}^{(i, j)}-\mathcal{I}_{t_{\ell+1}}^{(i, j)}\right| .
$$

Multi-date change measurement is performed by considering the absolute value of the combination of two-by-two comparison. At a given location $(i, j)$, the change amplitude between observations acquired at times $t_{\ell}, t_{\ell+1}, \ldots, t_{\ell+N}$ is computed by:

$$
\delta^{(i, j)}\left(t_{\ell} \rightarrow t_{\ell+N}\right)=\left|\sum_{k=0}^{N-1} \beta_{k}\left(\mathcal{I}_{t_{\ell+k}}^{(i, j)}-\mathcal{I}_{t_{\ell+k+1}}^{(i, j)}\right)\right| .
$$

Those two kinds of measure (bi-date and multi-date) will be denoted to as $\delta^{(i, j)}$ without lack of generality in the decision making step. It is assumed here that the change measure (bi-date and multi-date) may be modeled by:

$$
\delta^{(i, j)}=\varepsilon^{(i, j)} \mathcal{C}^{(i, j)}+\eta^{(i, j)},
$$

where $\mathcal{C}^{(i, j)}$ stands for the change intensity between acquisition dates, at the location $(i, j)$. This change can be present or absent: $\varepsilon^{(i, j)}=1$ in presence of change and $\varepsilon^{(i, j)}=0$ when no change occurs for pixel $(i, j) . \eta$ stands for noise in the estimation of the change measure.

(iii) Detection of change areas, from the map $\delta^{(i, j)}$ (not detailed here). It is performed by considering that changes are more likely to be absent $\left(\varepsilon^{(i, j)}=0\right)$ than present $\left(\varepsilon^{(i, j)}=1\right.$ ). This sparse assumption (when reasonnable) makes it possible the use of a non-parametric threashold-based decision making in the presence of $\varepsilon$ with no consideration to the shape of $\mathcal{C}$.

\section{MULTI-DATE PROGRESSIVE CHANGE INDICATOR}

Extension of bi-date change measure to multi-date may be investigated by extending bi-date measures of eq. (2) to a combination of elementary detectors to yield eq. (3).

\subsection{Multi-temporal filter definition}

The multitemporal change measure may take into consideration positive or negative changes so that the modified two-by-two measure is becoming as following:

$$
\delta^{(i, j)}\left(t_{\ell}, t_{\ell+1}\right)=\mathcal{I}_{t_{\ell}}^{(i, j)}-\mathcal{I}_{t_{\ell+1}}^{(i, j)} .
$$




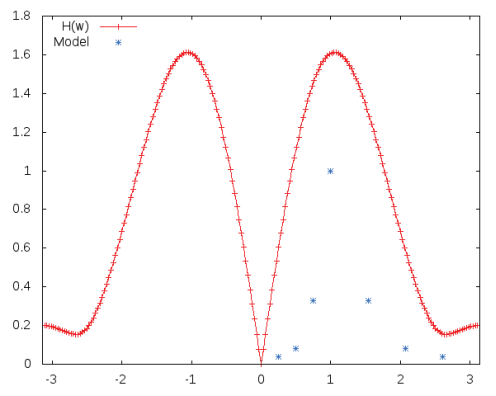

Fig. 1. Typical example of frequency response of filter (7) with a central pulsation of $\omega_{0}=\pi / 3$, with a filter length of $N=4$.

Then, the progressive change measure in-between dates $t_{\ell}$ and $t_{\ell+N}$ may be defined by considering a combination of $N$ twoby-two measures, through a $N$-tap filter:

$$
\delta^{(i, j)}\left(t_{\ell} \rightarrow t_{\ell+N}\right)=\sum_{k=0}^{N-1} \beta_{k}\left(\mathcal{I}_{t_{\ell+k}}^{(i, j)}-\mathcal{I}_{t_{\ell+k+1}}^{(i, j)}\right) .
$$

The progressive change measure of (6) may be interpreted to as a simple linear filter that transforms input samples $x[\ell]$ to outputs $y[\ell]$ as:

$$
y[\ell]=\sum_{k=0}^{N-1} \beta_{k}(x[\ell+k]-x[\ell+k+1])
$$

which helps in the evaluation of the parameters $\beta_{k}$ for progressive change detection purpose.

\subsection{Multi-temporal filter characterization and simulation}

From eq. (7), a $z$-transform analysis may yield to the harmonic analysis as well as the characterization of the response to specific inputs. As illustrated in Fig. 1, this filter may be built as a band-pass filter which is the aim of progresssive change detection (here, the central pulsation is choosen to be $\omega_{0}=\pi / 3$ which corresponds to a 6-month period caracterization through a regular time series of ERS images). Since, no analytical expression exist to evaluate the $\beta_{k}$ coefficients, a simulated annealing procedure as been implemented in order to estimate the $\beta_{k}$ coefficients to turn the behavior of the filter into a pass-band filter of central pulsation $\omega_{0}$.

Analysis of the $z$-transform indicates also the behavior of the filter when the input is given. Then, it can be shown that the response to a ramp is an edge; the response of an exponential is a weighted combination of shifted exponentials. The analytical expressions are not given here for space consideration but illustrated through noisy simulations in the next section.

Simulated time series have been built from a temporal behavior of reflectance; speckle noise has been multiplied to those homogeneous images according to a sampling of Gamma law. The reflectance value is depending on time parameter and its behavior is built to characterize a step, a ramp, a exponential change and a parametric model coming from [4] that characterizes the evolution of $\sigma_{0}$ from a rice crop. Simulation Curves confirm theoretical results that state that the signature of a ramp is an edge and the one of an exponential is an exponential. The crop evolution may also be characterized through a specific response that seems to be the derivative of the model (in fact, it is a combination of a ramp and a step). The simulations show also that in presence of noise, Recursive Median filtering is still capable to represent shade of evolutions.

\section{EXPERIMENTAL RESULTS}

This section provides experimental results on a time series of SAR images acquired over the Arcachon bay in France. 28 images were acquired by using ERS-SAR or ENVISAT-ASAR sensor from 2002 to 2006; nevertheless images of similar pass (ascendant or descendant orbit) and equivalent track may be used jointly so that no geometrical effects may be considered to as change (nevertheless, there is no need to follow interferometric conditions.) Change detection is performed by using the procedure described in Section 1.3. This procedure involves Recursive Median filtering of the observations as in eq. (1), and progressive change measure, based on the filter defined in (6). 


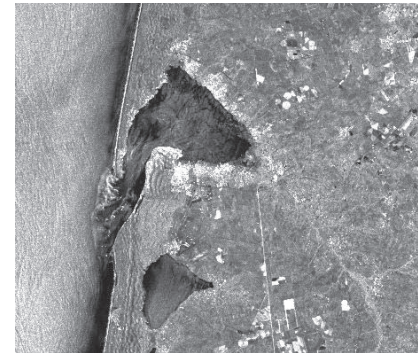

(a) ERS $I_{2}$, June 6, 2003

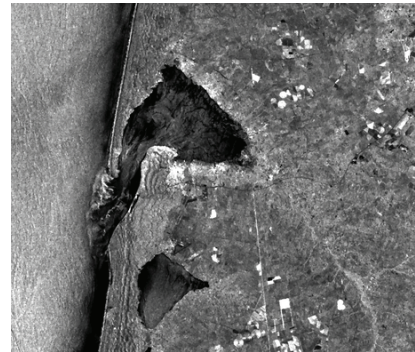

(b) ERS $\mathcal{I}_{2}$, June 6, 2003

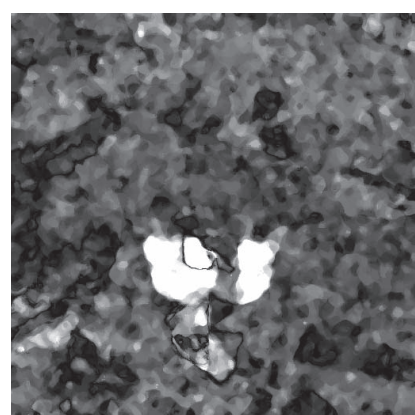

(c) Detection through eq. (2)

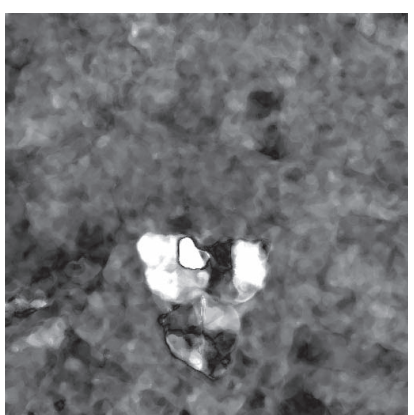

(d) Detection through eq. (3)

Fig. 2. One of the images in the SAR time series acquired over Arcachon bay. (a) original image, (b) RM filtered image. Differences between abrupt (c) and progressive change detection (d) in a typical ROI in the south of the city of Arcachon. Some of the fields does not show the same temporal behavior. (C) CNES

Then, every image $I_{\ell}$ of the time series is processed by using the RM algorithm to obtain $\mathcal{I}_{\ell}=\mathrm{RM}_{7 \times 7}\left(I_{\ell}\right)$, by using a sliding window of size $7 \times 7$. Next, multi-temporal filter of (6) is applied by using a filter of length $N=4$.

Fig. 2-(a) and (b) show one of those image. We will focus on the year 2003 and on a group of fields located in south of the city of Arcachon. Fig. 2-(c) and (d) shows two kind of change indicator: Fig. 2-(c) is issued from the bi-date detection of eq. (2) while Fig. 2-(d) shows the multi-temporal characterization of eq. (3). A deeper analysis will be added in the final article to show the accuracy in the discrminination of specific temporal profile, especially on fields area.

\section{CONCLUSION}

A model has been proposed for change detection in a time series of SAR images. The main idea is to turn the change detection procedure into the framework of detecting an unkonwn signal into a Gaussian noise, even if the present paper mainly focus on the change indicator instead of the detection itself.

The recurcive median filtering has been used to perform an appropriate regularization of the initial observations, despite the presence of speckle noise. That leads to an efficient algorithms that is not time demanding which yields piecewise constant images. Regularized image differencing performs a better change measure that the CKL-based change indicator, especially when using small size of sliding window, i.e. in high resolution change detection.

A combination of two-by-two image comparisons has been proposed in order to better characterize progressive changes in the time series. It appears to yield key information in the temporal behavior of the scene. Nevertheless, this progressive change indicator is assuming a regular sampling in time since it is associated to a linear filtering approach. Missing value is a time series does not affect dramatically this kind of temporal analysis. Nevertheless, when the time sampling is not regular (which is often the case in remote sensing time series) is not possible to interpret the shape of the output as having a bio-physical meaning to characterize the temporal behavior of the time series. A specific analysis of irregular time sampling in time series should be engaged.

\section{References}

[1] J. Inglada and G. Mercier, "A New Statistical Similarity Measure for Change Detection in Multitemporal SAR Images and its Extension to Multiscale Change Analysis," IEEE Trans. Geosci. Remote Sensing, vol. 45, no. 5, pp. 1432-1446, May 2007.

[2] Y. Bazi, L. Bruzzone, and F. Melgani, "Automatic identification of the number and values of decision thresholds in the log-ratio image for change detection in sar images," IEEE Geosci. Remote Sensing Letters, vol. 3, no. 3, pp. 349-353, July 2006.

[3] S. Alliney, "Recursive median filters of increasing order: A variational approach," IEEE Trans. Signal Processing, vol. 44, no. 6, pp. 1346-1354, June 1996.

[4] T. Kurosu, M. Fujita, and K. Cjiba, "Monitoring of rice crop growth from space using the ERS-1 C-band SAR," IEEE Trans. Geosci. Remote Sensing, vol. 33, no. 4, pp. 1092-1096, July 1995. 